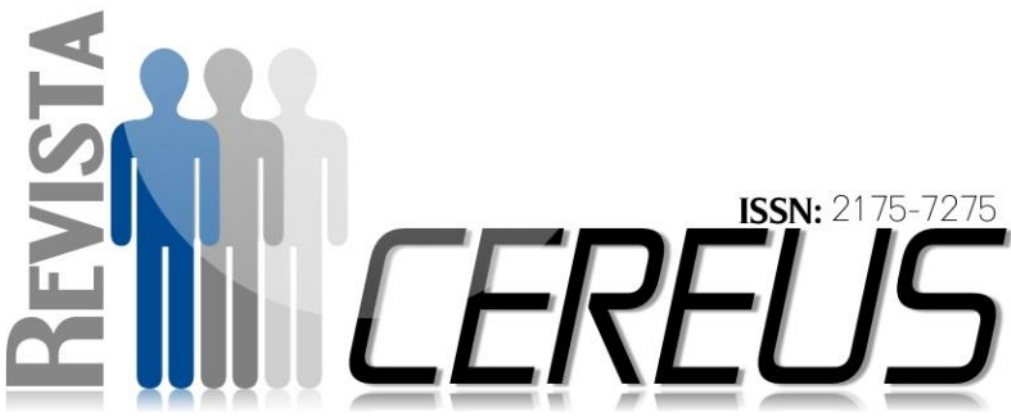

\section{MANEJO DE RESÍDUOS SÓLIDOS DE SAÚDE, EM DUAS UNIDADES DE SAÚDE EM PALMAS - TOCANTINS: BASES PARA O GERENCIAMENTO}

RIBEIRO, Marildo de Sousa ${ }^{1}$

CASTRO, José Gerley Diaz ${ }^{2}$

\section{RESUMO}

Os Resíduos de Serviço de Saúde (RSS), devido às suas características tóxicas e/ou patogênicas, constituem uma inquietação para a sociedade e para o meio ambiente. Todo gerador deve elaborar um Plano de Gerenciamento de Resíduos de Serviços de Saúde - PGRSS. O trabalho objetivou contribuir com o gerenciamento de resíduos de saúde no município de Palmas mediante a implantação do Manejo correto dos RSS, em duas Unidades de Saúde do município. Adotou-se o desenho de pesquisa do tipo intervenção, com caráter propositivo. Análise estatística para o efeito da capacitação sobre a implantação do PGRSS foi analisado mediante 0 teste não paramétrico dos sinais (AYRES et al., 2007) considerando as duas datas de observação (22/02/2016 - ANTES e 02/06/2016 - DEPOIS).

\footnotetext{
${ }_{1}^{1}$ Programa de Mestrado Ciências da Saúde - UFT Email: marildosousa@hotmail.com

${ }^{2}$ Docente do Programa de Mestrado Ciências da Saúde - UFT
} 
Verificado o resultado do teste dos sinais para o caso da implantação do PGRSS para o CEO704S, este é muito significativo (pbinomial $=0,0002$ ) e o poder do teste igual a 0,9993 . Resultados similares foram encontrados para POLI108S ( $p<0.0001)$ e poder de 0,9998 . O que indica a importância da qualificação dos servidores para a implantação do Programa nas duas unidades. Identificou-se que o manejo dos resíduos das unidades não estava sendo operacionalizados de forma adequada nas diferentes etapas do processo de gerenciamento, e que o produto desta pesquisa atendeu o esperado que foi a implantação do PGRSS nestes serviços, com índice de conformidade igual ou superior a $85 \%$ conforme o estabelecido pelas resoluções vigentes no país, no final da pesquisa.

Palavras chave: Hospital. Rejeito. Gerenciar.

SOLID WASTE MANAGEMENT OF HEALTH IN TWO HEALTH UNITS IN PALMAS - TOCANTINS: BASES FOR MANAGEMENT

\section{ABSTRACT}

Waste Health Service (RSS), due to its toxic and/or pathogenic characteristics, are a concern for society and the environment. Every generator should prepare a Plan of Health Services Waste Management - PGRSS. The study aimed to contribute to the health of waste management in the city of Palmas by implementing the right of RSS Management in two municipal health units. Adopted the research design of the intervention type, with propositional character. Statistical analysis for the effect of training on the implementation of PGRSS was analyzed by the nonparametric sign test (Ayres et al., 2007) considering the two observation dates (22/02/2016 - 06/02/2016 BEFORE and - AFTER). Checked 
the signs test results in the case of PGRSS deployment to the CEO704S, this is very significant (pbinomial $=0.0002$ ) and the power of the test equal to 0.9993. Similar results were found for POLI108S ( $p<0.0001)$ and power 0.9998. This indicates the importance of qualification of servers for the Program implementation in the two units. It was identified that the management of waste from the units were not being operated properly in the different stages of the management process, and that the product of this research has met the expected was the implementation of PGRSS these services, compliance index equal to or greater $85 \%$ as established by the resolutions in force in the country at the end of the research.

Keywords: Hospital. Reject. Manage. 


\section{INTRODUÇÃO}

Segundo a Organização Mundial da Saúde, cerca de $1 \%$ dos resíduos urbanos gerados são equivalentes aos resíduos de serviços de saúde (RSS) e dependendo da complexidade pode chegar até a $3 \%$ (JACOBI \& BESEN, 2011). Estes são gerados nos ambientes que prestam serviços aliados a saúde, ou seja, hospitais, postos de saúde, consultório odontológico, farmácias, laboratórios clinicas médicas e veterinárias.

Os RSS, devido às suas características tóxicas e/ou patogênicas, constituem um problema para a sociedade e para o meio ambiente. Para Pereira (2013) a preocupação com os resíduos gerados pelas diversas atividades humanas, em especial os advindos dos serviços de saúde, tem seu marco legal no Brasil, a partir das publicações da RDC 306/2004, da Agência Nacional do Meio Ambiente - ANVISA, RDC nํ 358/2005 do Conselho Nacional do Meio Ambiente - CONAMA, visto que estabeleceram a harmonização entre os órgãos regulatórios a respeito RSS, e que ambas transferiram a responsabilidade do manejo para os geradores.

A gestão adequada dos RSS aumenta este desafio. Para Silva e Bonfada (2012), o gerenciamento inadequado dos RSS causa impactos ambientais, tais como contaminações, altos índices de infecções hospitalares podendo levar a endemias ou mesmo epidemias, devido a contaminações do solo, ar e lençóis freáticos pelos diferentes tipos de resíduos de serviços de saúde.

Para Caetano e Gomes (2013), há um gama de exigências legislações brasileiras refere ao gerenciamento dos RSS, como resoluções de diretorias de colegiados (RDC), leis, e portarias ministeriais. Porém, na prática, estas nem sempre são cumpridas devido, principalmente, à falta de priorização destas ações pelos gestores, à falta de recursos da instituição, controle e fiscalização dos órgãos competentes. Em decorrência disto, grande parte dos estabelecimentos do país não gerenciam adequadamente seus resíduos, pondo em risco a saúde pública e contaminando 0 meio ambiente. Portanto Pereira (2011), já alertava que esta gama de exigência remete para cada gerador, ou seja, 
cada estabelecimento de saúde, a responsabilidade de gerenciar os resíduos gerados.

Todo gerador deve elaborar um Plano de Gerenciamento de Resíduos de Serviços de Saúde - PGRSS, baseado nas características dos resíduos gerados e nas suas classificações e estabelecendo as diretrizes de manejo dos RSS.

O Gerenciamento dos RSS constitui-se em um conjunto de procedimentos de gestão, planejados e implementados a partir de bases científicas e técnicas, normativas e legais, com o objetivo de minimizar a produção de resíduos e proporcionar aos resíduos gerados, um encaminhamento seguro, de forma eficiente, visando à proteção dos trabalhadores, a preservação da saúde pública, dos recursos naturais e do meio ambiente. Este deve abranger todas as etapas de planejamento dos recursos físicos, dos recursos materiais e da capacitação dos recursos humanos envolvidos no manejo dos RSS (BRASIL 2004, BRASIL 2005).

Esta responsabilidade também é confirmada em 2010 quando foi promulgada e lei 10.305 de 02/08/2010 que institui a Política Nacional de Resíduos Sólidos, determinando em seu artigo 20 que os serviços de saúde estão sujeitos a elaboração do PGRSS.

A Rede de Saúde do município de Palmas constitui de Unidades de Saúde da Família (USF); Policlínicas; Centro de Consultas Especializadas de Palmas (CECEP); Centro de Atendimento Psicossocial (CAPS) e CAPS II- Álcool e Drogas; Núcleo de Atendimento Henfil (para DST's e AIDS); Centro de Saúde Sexual e Reprodutivo; Centro de Especialidades Odontológicas (CEO); Unidades de Pronto Atendimento 24h/dia (UPA's), de forma integrada ao SAMU 192 (Serviço Móvel de Urgência e Emergência); Centro de Controle em Zoonoses (CCZ); Redes de Farmácias Municipais, (PALMAS, 2016).

Por esta complexidade da prestação de serviço municipal, muitos fatores vêm contribuindo para o aumento da geração de resíduos, impactando também, na saúde ambiental, tais como: o crescimento da complexidade médica, aumento do número de pessoas com acesso aos atendimentos em saúde, municipalização das ações e programas em saúde.

Segundo Pereira (2013) deve ser considerado o impacto dos RSS no ecossistema e seu significado 
epidemiológico no contexto da saúde pública, e também questionar-se: como esse assunto é tratado nesses serviços? Existe uma política institucional sistematizada para o seu gerenciamento? A questão dos RSS é de uma relevância inquestionável tanto nos contextos da segurança dos profissionais de saúde e da preservação e conservação do meio ambiente como no âmbito da construção de novos paradigmas de atenção à saúde.

Neste contexto a proposta de trabalho vislumbrou-se desenvolver em duas Unidades de Saúde da rede, uma Policlínica e um Centro de Especialidade Odontológica, um Projeto Piloto de Plano Gerenciamento de Resíduos de Serviço de Saúde (PGRSS) que atenda as necessidades técnicas e legais da gestão de resíduos, desde a geração à disposição final.

Em visita as Unidades de Saúde, em que foi realizado este trabalho, Policlínica da 108 Sul (POLI 108S) e Centro Especializado em Odontologia da 704 Sul (CEO 704S), ambas em Palmas, constatou-se a inexistência do PGRSS, remetendo a uma grande necessidade de elaboração destes documentos, como como a implantação do mesmo, evidenciou-se que o caminho seria contemplar um arrojado Plano de Educação Continuada sobre a temática, buscando com isso o envolvimento de todos os servidores.

O PGRSS é um documento que atende a legislação vigente, que comprova a capacidade de uma Unidade de Saúde de gerir todos os resíduos por ela gerados. $\mathrm{O}$ documento expressa intenção de se ter a segurança de que as atividades prestadas pela organização, a uma determinada comunidade, são controladas para evitar grandes impactos ambientais e as devidas consequências para a saúde pública.

\subsection{OBJETIVOS}

\section{GERAL:}

Contribuir com o gerenciamento de resíduos da saúde no município de Palmas mediante a implantação do Manejo correto de Resíduos Sólidos de Saúde, em duas Unidades de Saúde do município.

\section{ESPECÍFICOS:}

Identificar as atividades desenvolvidas em cada Unidade e conhecer os resíduos gerados.

Identificar o nível de adequação de cada Unidade, quanto às normas 
técnicas e legislação vigente, referente ao Gerenciamento de Resíduos.

Conhecer o nível de informação dos funcionários referente à temática: Gerenciamento dos Resíduos de Serviços de Saúde.

\section{METODOLOGIA}

O trabalho foi realizado em duas Unidades de saúde de Palmas: POLI $108 S$ e CEO 704S.

A pesquisa adotou o método qualitativo com desenho de pesquisa do tipo intervenção, onde procurou explorar as causas que condicionam a atual situação do manejo de resíduos de cada unidade, com caráter propositivo, baseado em Lacerda et al. (2015).

O projeto foi submetido e aprovado pelo Comitê de Ética em Pesquisa do Centro Universitário Luterano de Palmas - ULBRA, com aprovação número 1.188.907, de 28/08/2015.

Todos os servidores das unidades de saúde que foram convidados a participar voluntariamente da pesquisa, foram todos instruídos e assinaram Termo de Consentimento Livre e Esclarecido.
Qualificar profissionais de cada Unidades para que o mesmo tenha condições de elaborar e implantar o Plano de Gerenciamento de Resíduos de Serviços de Saúde.

Incialmente levantou-se os dados de cada unidade de saúde, através de uma ferramenta (questionário fechado) de levantamento de dados, validada pelo Ministério da Saúde - Questionário de Diagnóstico de Aspectos Ambientais e Manejo de Resíduos do Serviço de Saúde (BRASIL, 2012a).

$\mathrm{Na}$ segunda etapa do trabalho, utilizou a técnica de observação participativa. Para 0 diagnóstico situacional do gerenciamento de resíduos em cada Unidade de Saúde, observou-se as rotinas de trabalho, com ênfase ao manejo de resíduos e processos de lavagem e esterilização por três dias alternados, nos turnos matutino e vespertino. $O$ registro destes dados, foram ordenadamente registrados em "diário de campo" e fotografias.

A terceira etapa admitiu-se a utilização de um checklist, - Roteiro de 
Avaliação da Implantação do PGRSS.

Pactuou-se com os responsáveis de cada unidade de saúde, quais os itens, relacionados ao gerenciamento dos resíduos (baseado em boas práticas e legislação vigente) sofreriam intervenção direta com o trabalho, e que a reavaliação do cenário ocorreria após um período decorrido de 100 dias, sem a presença direta da assessoria do pesquisador. A utilização deste roteiro permitiu quantificar a porcentagem de adequação ocorrida nas instituições após a realização da pesquisa. Nesta pesquisa a análise estatística para o efeito da capacitação sobre a implantação do PGRSS foi analisado mediante o teste não paramétrico dos sinais (AYRES et al., 2007) considerando as duas datas de observação (22/02/2016 - ANTES e 02/06/2016 - DEPOIS). Para o Erro Tipo I, foi considerado um valor de $5 \%$.

Procedeu-se com um programa de qualificação dos servidores,

\section{RESULTADOS}

Os resultados da pesquisa mostrados no Quadro 1, destaca-se que dos cinco itens avaliados no quesito aspectos gerenciais do RSS, $100 \%$ estavam não conformes, contemplando todas as etapas do manejo de resíduos. Através de debate expositivo, facilitado pelo pesquisador. Esta etapa de qualificação foi repetida várias vezes em cada Unidade de saúde; três vezes no CEO $704 S$ e 08 vezes na POLI 108S, objetivando alcançar um maior número de servidor possível, sem interrupção das atividades.

Foram elaborados os Procedimentos Operacionais Padrão (POP) de Lavagem e Esterilização de matérias (laboratoriais e odontológicos) e POP Limpeza e Desinfecção de Áreas. Para cada protocolo elaborado, treinou-se a equipe específica que executaria.

Por último foi elaborado e disponibilizado á cada Unidade de saúde, o Plano de Gerenciamento de Resíduos de Serviço de Saúde (PGRSS) em consonância com as legislações vigentes.

equivalência encontrada nas duas unidades. Enquanto que os aspectos ambientais e de saúde do trabalhador, variam entre conformes e não conformes, no início do trabalho. 
Quadro 1 - Questionário de diagnóstico - aspectos ambientais e manejo de resíduos do serviço de saúde

\begin{tabular}{|c|c|c|}
\hline \multirow[b]{2}{*}{ ITENS OBSERVADOS } & \multicolumn{2}{|c|}{ UNIDADES DE SAÚDE } \\
\hline & $\begin{array}{l}\text { POLICLÍNICA DA } 108 \\
\text { SUL }\end{array}$ & $\begin{array}{l}\text { CENTRO } \\
\text { ESPECIALIDADES } \\
\text { ODONTOLÓGICAS } \\
\text { (CEO) DA } 704 \text { SUL }\end{array}$ \\
\hline \multicolumn{3}{|c|}{ ASPECTOS AMBIENTAIS } \\
\hline $\begin{array}{l}\text { Fornecimento de Água tratada e } \\
\text { conexão com a rede coletora de esgoto. }\end{array}$ & + & + \\
\hline $\begin{array}{llll}\text { ldentificação } & \text { e } & \text { Segregação } & \text { dos } \\
\text { resíduos } & & & \\
\end{array}$ & - & - \\
\hline $\begin{array}{lccc}\text { Container } & \text { e } & \text { lixeiras } & \text { para } \\
\text { acondicionamento dos resíduos } & \end{array}$ & - & - \\
\hline $\begin{array}{llll}\text { Disposição final dos } & \text { resíduos } \\
\text { adequado. o } & & & \\
\end{array}$ & + & + \\
\hline Abrigo de resíduos & - & + \\
\hline $\begin{array}{l}\text { Higienização das áreas (pisos, portas, } \\
\text { janela e balcões e pias) }\end{array}$ & - & - \\
\hline \multicolumn{3}{|c|}{ ASPECTOS GERENCIAIS NA INSTITUIÇẦ } \\
\hline Responsável formal pelo PGRSS & -4 & - \\
\hline PGRSS implantado & - & - \\
\hline Protocolos de trabalho - POP & - & - \\
\hline Possuem alvará sanitário & - & - \\
\hline $\begin{array}{l}\text { Programa de treinamento periódico dos } \\
\text { servidores em gestão ambiental }\end{array}$ & - & - \\
\hline \multicolumn{3}{|c|}{ ASPECTOS RELACIONADOS A SAÚDE DO TRABALHADOR } \\
\hline Vacina & + & + \\
\hline $\begin{array}{l}\text { Uso de EPl's (calçados, luvas, } \\
\text { máscaras e gorros e jaleco impermeável } \\
\text { quando necessário) }\end{array}$ & - & - \\
\hline
\end{tabular}

Constata-se que nas duas unidades os aspectos gerencias, são os que mais necessitavam de ações, dentro da pesquisa que pudesse conduzir as unidades para o pleno atendimento das boas práticas e legislações vigentes. As condições físicas e de insumos (containers, lixeiras e saneantes), eram favoráveis, expressando que o desafio maior para regularizar a situação da Gestão Ambiental era apenas em sua maioria de caráter gerencial. Este diagnóstico serviu de balizador para as demais etapas do trabalho.

Verificou-se no decorrer da pesquisa que a disposição final dos resíduos, produzidos pelas duas 
unidades de saúde, ocorrem de forma apropriada e atende a legislação vigentes. Os resíduos comuns, grupo D, são encaminhados para o aterro sanitário licenciado. Os resíduos do grupo A, B e E, são incinerados em empreendimento licenciados e as cinzas são enviadas para aterro sanitário Classe I, específico para esta disposição, também licenciado. Foram disponibilizados, pelas empresas responsáveis, às unidades de saúde, os seguintes documentos: Licença para do transportes dos resíduos (perigosos e não perigosos); Licença de operação da empresa de incineração; licença do aterro certificados de descarte das cinzas. Todos estes documentos estão em poder das unidades onde foram desenvolvidas a pesquisa.

$\mathrm{Na}$ observação das rotinas de trabalho, agora especificamente no que refere ao manejo de resíduos, limpeza, lavagem e esterilização, permitiu a evidenciar a ocorrência dos principais vícios e erros, os quais aqui mencionados: Uso inadequado dos saneantes (diluições diferentes das recomendadas pelos fabricantes), erro na execução de higienizações (a limpeza não precedida a descontaminação) e estilizações (processos não validados), transporte inadequados dos resíduos gerados (sem a utilização de carrinhos), contrariando 0 preconizado pelo Manual de Segurança do Paciente em serviços de Saúde: Limpeza e Desinfecção de Superfícies, do Ministério da Saúde (BRASIL 2012b). Verificou-se o uso indevido dos EPI's. Esta prática contraria 0 que é preconizado pela NR 32, colocando em ameaça a segurança e a saúde do trabalhador (BRASIL, 2005).

$\mathrm{Na}$ segunda etapa da observação participativa, foi possível acompanhar e ao mesmo tempo desenvolver protocolos de trabalho, compatíveis com a legislação vigente e instruções de órgãos reguladores brasileiros, para a execução destas atividades.

Desenvolveu-se um curso de capacitação em gerenciamento de resíduos, com duração de 2 horas. Os temas abordados foram: gerenciamento de resíduos de serviços de saúde (conceitos básicos de resíduos, legislação, tipos de resíduos de serviços de saúde (RSS), segregação e acondicionamento, tratamentos dos RSS, disposição final); Plano de Gerenciamento de Resíduos de Serviço de Saúde (PGRSS); 
Lavagem e Esterilização de material laboratorial e odontológico; Limpeza e desinfecção de ambientes hospitalares. Assim estas unidades passam atender o estabelecido pela legislação vigente, RDC's ANVISA 306/04, que determina a obrigatoriedade do serviço em prover a capacitação e o treinamento inicial e de forma continuada para o pessoal envolvido no gerenciamento de resíduos. O Gráfico 01 , mostra o número de servidores, em de cada unidade, que participou da pesquisa através da assinatura dos Termos de Consentimento Livre Esclarecido e a quantidade de participantes dos cursos.

O estudo mostrou que havia um baixo percentual de qualificação dos trabalhadores que atuam nas unidades de saúde, na temática gerenciamento de resíduos. No levantamento de perfil dos servidores de cada unidade, realizado no início da pesquisa, observa-se que, 74\% (CEO 704S) e 97\% (POLI 108S), declararam nunca terem sido instruídos em gerenciamento de resíduos, conforme Gráfico 02.
Gráfico 01 - Participantes da pesquisa e cursistas nas duas Unidades de Saúde

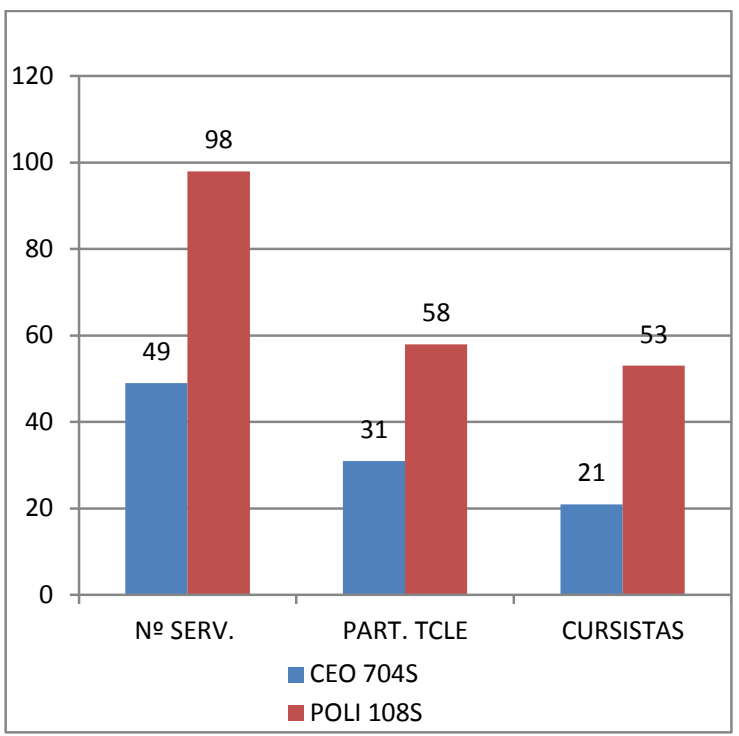

Gráfico 02 - Porcentagem de servidores que já haviam realizado cursos

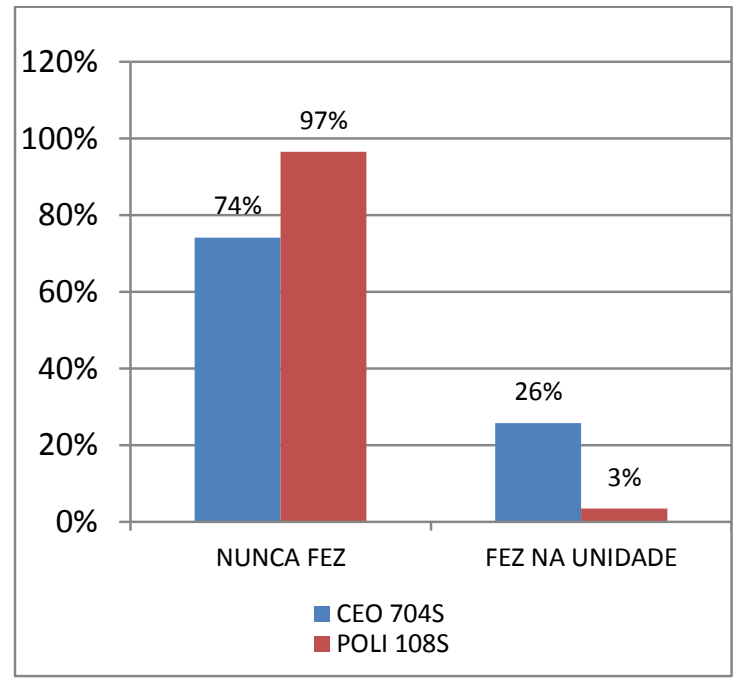


A limpeza e a higienização são fundamentais para proporcionar um entorno saudável e livre de agentes causadores de doenças, como bactérias e fungos que persistem em ambientes hospitalares, possibilitando doenças nosocomiais. Cada tipo de ambiente de unidades de saúde requer técnicas de limpeza específicas, produtos adequados e mão de obra treinada. No início da pesquisa, constatou-se que não havia nenhum protocolo de trabalho (POP) referente aos processos de manejo de resíduos, lavagem e esterilização e limpeza e desinfecção.

Neste sentido, com 0 desenvolvimento da pesquisa, foi elaborado para cada serviço os seguintes manuais, protocolos e planilhas de registo:

Plano de Gerenciamento de Resíduos de Serviços De Saúde PGRSS;

\section{Procedimento Operacional} Padrão - Lavagem de Áreas e Superfícies - POP - LAS.

Procedimento Operacional Padrão - Lavagem de Instrumentos Cirúrgicos e Odontológicos - POP LICO.

Planilha de Registro de Limpeza de Piso - PRLP
Planilha de Avaliação Setorial: Segregação e Acondicionamento dos Resíduos - PAS-SAR.

Em um ambiente em que o risco de contaminação é potencialmente maior, é fundamental seguir corretamente os protocolos de limpeza e desinfecção. Muitas doenças são causadas por agentes infecciosos e, em hospitais e clínicas, esses microrganismos podem contaminar superfícies e equipamentos que são frequentemente manuseados por profissionais que ali atuam. A correta forma de executar a sanitização dos ambientes e materiais, contribui para o controle da proliferação de doenças e da contaminação cruzada, assegurando o bem-estar de quem oferece e se beneficia dos serviços de saúde (BRASIL, 2012b).

As etapas do gerenciamento dos RSS, que vão deste a identificação, segregação, acondicionamento, transporte interno, armazenamento externo, coleta e transporte externo, tratamento até a disposição final, precisam de um ordenamento nas unidades de saúde objetivando atender as boas práticas e legislação vigente (STEHLING, 2013).

Assim, no Quadro 2, apresenta a ferramenta, Roteiro de Avaliação da 
Implantação do PGRSS, utilizada como forma de mensurar a evolução de adequação, em percentual, de cada instituição, antes e depois da pesquisa, no que refere ao processo de gerenciamento dos seus resíduos gerados.

Nesta pesquisa a análise estatística para o efeito da capacitação sobre a implantação do PGRSS foi analisado mediante 0 teste não paramétrico dos sinais (AYRES et al., 2007) considerando as duas datas de observação (22/02/2016 - ANTES e 02/06/2016 - DEPOIS).

Quando verificado o resultado do teste dos sinais para o caso da implantação do PGRSS para o CEO 704 S, este é muito significativo (pbinomial $=0,0002)$ e o poder do teste igual a 0,9993. Resultados similares foram encontrados para POLI 108 S ( $p$ $<0.0001$ ) e poder de 0,9998. O que indica a importância da qualificação dos servidores para a implantação do PGRSS nas duas unidades de saúde. Nos dois locais, verificou-se que a evolução das adequações do gerenciamento, relacionados aos aspectos gerenciais da unidade nas instituições (Quadro 1), inadequações corrigidas com qualificação e instrução da equipe de trabalho.
O Quadro 2 mostra ainda que a evolução de adequações nas duas unidades de saúde foi muito grande. $\mathrm{O}$ CEO 704S foi a unidade que melhor se adequou ao gerenciamento de resíduo até o final da pesquisa, mas nas duas unidades, o resultado da pesquisa, conduziu a índices maior ou igual a $85 \%$ de adequação, como mostra o Gráfico 03.

O item que não teve sua adequação concluída nas duas unidades, até o encerramento desta pesquisa foi a aquisição de carrinhos para o escoamento dos resíduos, do ponto de geração até 0 abrigo, mostrado no Quadro 02. Embora o processo de aquisição destes equipamentos, teve seu inicio, como um produto da pesquisa, todavia as compras dentro das organizações públicas demandam tempo, fator determinante para a morosidade desta adequação. A utilização de carrinho para o escoamento dos resíduos é um item obrigatório, determinado pelas RDC's ANVISA 306/04 e CONAMA $358 / 04$, pois confere maior segurança para o operador das ações e para os transeuntes do espaço, visto que unidades de saúde possuem um elevado fluxo de pessoas constantemente. 
A POLI 108S, teve $85 \%$ de adequação até o final desta pesquisa, a impossibilidade da adequação de dois fatores, a mais que a outra unidade, colabora para este resultado final. No início da pesquisa, detectou-se que o abrigo de resíduos e a central de lavagem de material, eram inadequados. Não foram somados esforços, para a solução destes dois problemas, vista que esta unidade está na eminência de ser transferida para sua sede definitiva, dotadas de áreas especificas e exclusiva para estas finalidades.

Vale destacar que a mudança física da unidade POLI 108S, não reduz a significância do trabalho ora realizado. Destaca-se que, a implantação de manual (PGRSS) e protocolos de trabalho, bem como o envolvimento de toda a força de trabalho, até então alcançado, é aplicável na organização, independente da dinamicidade física sofrida por esta.

De acordo com o Manual de Gerenciamento de Resíduos (BRASILIA, 2006), o PGRSS é um documento que aponta e descreve as ações relativas ao manejo de resíduos sólidos, que corresponde as etapas de: segregação, acondicionamento, coleta, armazenamento,

transporte, tratamento e disposição final, levando em consideração os riscos dos resíduos, as ações de proteção a saúde e ao meio ambiente.

Nesta análise devem ser
consideradas as características e
riscos dos resíduos, as ações de
proteção à saúde e ao meio
ambiente e os princípios da
biossegurança, empregar medidas
técnicas, administrativas e
normativas para prevenir
acidentes. O principal objetivo do
PGRSS não é apenas reduzir a
quantidade de resíduos com risco
biológico, mas também criar uma
cultura de segurança e do não
desperdício, além do envolvimento
coletivo. Em cada unidade de
saúde, o Plano deve ser feito em
conjunto com todos os setores,
definindo-se responsabilidades e
obrigações de cada um em
relação aos riscos. (LORENTZ,
2011, p. 19) Propondo atender esta carência dos serviços estudados, um dos produtos desta pesquisa foi a elaboração do PGRSS de cada unidade envolvida, que deverá ser revisado anualmente. Incialmente fez um levantamento de todos as atividades desenvolvidas em cada organização, para a partir disso identificar quais os tipos dos resíduos gerados, bem como propor a forma apropriada do gerenciamento destes. Vale destacar que são apontados nestes PGRSS, os fluxos de escoamento de resíduos, a forma correta de identificar e acondicionar de 
cada resíduo gerado, os indicadores de desempenho, bem com a conexão deste manual com os POP's de trabalho afins.

Para o monitoramento do PGRSS implantado, atendeu-se o preconizado pela RDC ANVISA 306/04 que determina:

4.2 - Compete ainda ao gerador de RSS monitorar e avaliar seu PGRSS, considerando;

4.2.1 - O desenvolvimento de instrumentos de avaliação e controle, incluindo a construção de indicadores claros, objetivos, autoexplicativos e confiáveis, que permitam acompanhar a eficácia do PGRSS implantado (RDC ANVISA 306/04).

Neste sentido, como produto desta pesquisa, e como parte do PGRSS implantado, desenvolve-se três indicadores.

Gestão à Vista - Indicadores que monitora mensalmente o processo de segregação, identificação e acondicionamento dos resíduos em seus pontos de geração, em todos os setores geradores, das unidades de saúde. Valor expresso em porcentagem de setores aprovados e reprovados no mês analisado, permitindo assim formar uma série histórica de adequação destes itens ao longo dos meses avaliados.

Qualificação dos servidores Indicador que monitora, ao longo do ano corrente, quantos servidores da organização foram qualificados e/ou requalificados em Gerenciamento de Resíduos. Valor expresso em porcentagem de servidores que passaram por qualificação, ano após ano dentro do universo de servidores que laboram na unidade.

Quantificação dos acidentes de trabalho - indicador que monitora ao longo do ano corrente, o número de acidente com servidores que trabalham diretamente com manejo de resíduos. Valor expresso em número absoluto.

Os indicadores geralmente são utilizados com propósito de se conhecer adequadamente uma situação existente, tomar decisões e monitorar sua evolução. Assim cada unidade de saúde, terá condições de monitorar o PGRSS implantado por esta pesquisa. 
Quadro 2 - Roteiro de Avaliação da Implantação do PGRSS

\begin{tabular}{|c|c|c|c|c|c|}
\hline \multirow{2}{*}{ № } & \multirow{2}{*}{ REQUISITOS } & \multicolumn{2}{|c|}{ CEO 704S } & \multicolumn{2}{|c|}{ POLI 108S } \\
\hline & & ANTES & DEPOIS & ANTES & DEPOIS \\
\hline 1 & $\begin{array}{l}\text { Existe responsável formal pelo Plano de } \\
\text { Gerenciamento de Resíduos de Serviço de } \\
\text { Saúde (PGRSS) da Unidade de Saúde? }\end{array}$ & - & + & - & + \\
\hline 2 & $\begin{array}{l}\text { Existe Plano de Gerenciamento de Resíduos de } \\
\text { Serviços de Saúde (PGRSS) escrito e aprovado } \\
\text { pelo Responsável pela Unidade de Saúde? }\end{array}$ & - & + & - & + \\
\hline 3 & $\begin{array}{l}\text { Existem Procedimento Operacional Padrão } \\
\text { (POP) necessários a operação do PGRSS: } \\
\text { Segregação, Coleta Interna dos Resíduos, } \\
\text { Limpeza? }\end{array}$ & - & + & - & + \\
\hline 4 & $\begin{array}{lll}\text { Existem Planilhas, Quadros } & \text { e } & \text { Tabelas } \\
\text { necessários a operação do PGRSS ? } & \end{array}$ & - & + & - & + \\
\hline 5 & $\begin{array}{l}\text { Existe fluxo de coleta de resíduos na Unidade de } \\
\text { Saúde }\end{array}$ & - & + & - & + \\
\hline 6 & $\begin{array}{l}\text { Existe simbologia padrão das lixeiras, de acordo } \\
\text { com a legislação, definida pelo PGRSS da } \\
\text { Unidade de Saúde. }\end{array}$ & - & + & - & + \\
\hline 7 & Existem Indicadores de Avaliação do PGRSS? & - & + & - & + \\
\hline 8 & $\begin{array}{l}\text { Existe evidências de treinamento da Equipe de } \\
\text { Coleta? }\end{array}$ & - & + & - & + \\
\hline 9 & $\begin{array}{l}\text { A equipe de Higienização, Lavagem e Estilização } \\
\text { utiliza os EPI adequados às atividades que } \\
\text { realizam? }\end{array}$ & + & + & - & + \\
\hline 10 & $\begin{array}{l}\text { Há evidências de treinamento dos funcionários } \\
\text { da Unidade em Gestão Ambiental da Unidade? }\end{array}$ & - & + & - & + \\
\hline 11 & $\begin{array}{l}\text { Tem licença atualizada da Empresa de } \\
\text { tratamento externo dos Resíduos? }\end{array}$ & - & + & - & + \\
\hline 12 & $\begin{array}{l}\text { Tem licença atualizada da Empresa de } \\
\text { transporte dos Resíduos? }\end{array}$ & - & + & - & + \\
\hline 13 & $\begin{array}{l}\text { A destinação final dos Resíduos é em aterro } \\
\text { licenciado? }\end{array}$ & + & + & - & + \\
\hline 14 & Existem lixeiras adequados e suficientes? & + & + & + & + \\
\hline 15 & $\begin{array}{l}\text { Existem carros adequados para a coleta de } \\
\text { resíduos? }\end{array}$ & - & - & - & - \\
\hline 16 & $\begin{array}{l}\text { Existe abrigo de resíduos externo conforme } \\
\text { legislação? }\end{array}$ & + & + & - & - \\
\hline 17 & 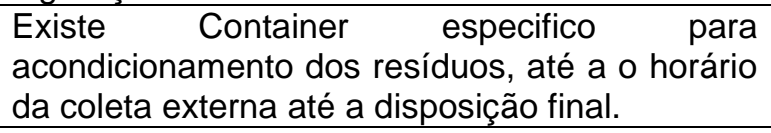 & + & + & + & + \\
\hline 18 & $\begin{array}{l}\text { Existe Central de Lavagem de } \\
\text { apropriada? }\end{array}$ & + & + & - & - \\
\hline 19 & $\begin{array}{l}\text { Existe sistema de validação do processo de } \\
\text { esterilização de material? }\end{array}$ & + & + & - & + \\
\hline 20 & $\begin{array}{l}\text { Existe Procedimento Operacional Padrão (POP) } \\
\text { para instruir as atividades executadas na Central } \\
\text { de Lavagem de Material? }\end{array}$ & - & + & - & + \\
\hline & PROCENTAGEM DE ADEQUAÇÃO & $35 \%$ & $95 \%$ & $10 \%$ & $85 \%$ \\
\hline
\end{tabular}


Gráfico 03 - Porcentagem de adequação do gerenciamento de resíduos nas duas Unidades.

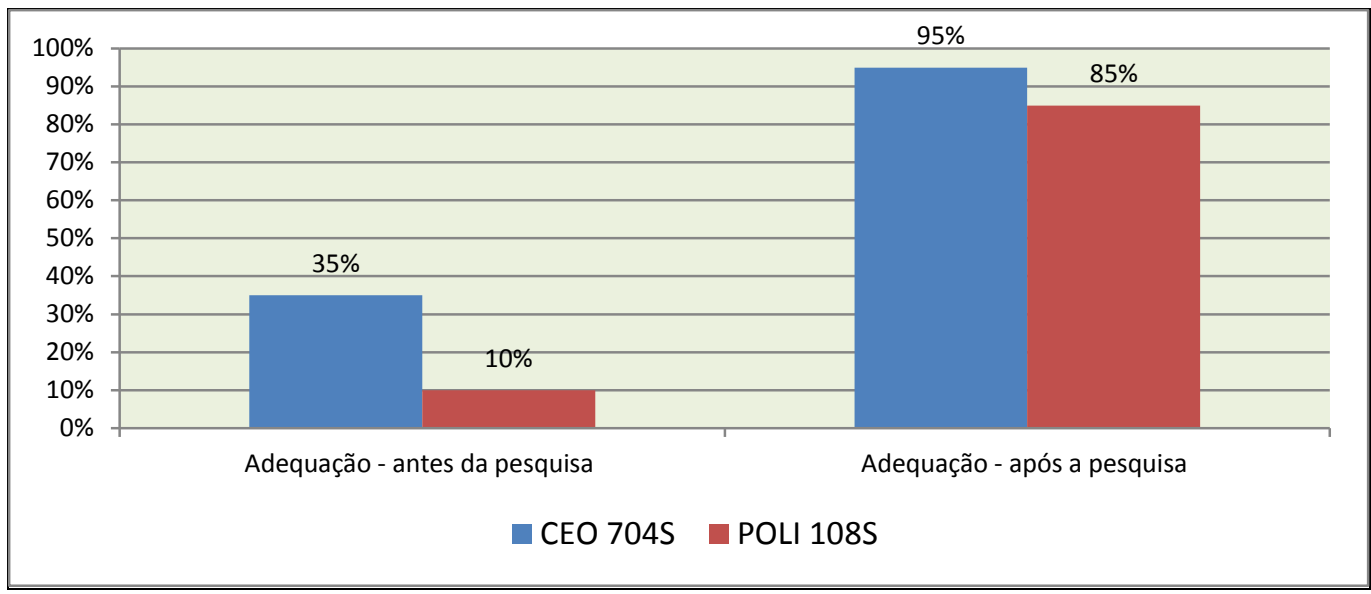

\section{CONSIDERAÇÕES FINAIS}

$\mathrm{Na}$ presente pesquisa identificou-se que 0 manejo dos resíduos das duas unidades não estava sendo operacionalizado de forma adequada nas diferentes etapas do processo, como, segregação, acondicionamento, identificação, coleta, armazenamento, transporte $\mathrm{e}$ disposição final, e que o produto desta pesquisa atendeu o esperado que foi a implantação do PGRSS neste serviço, com índice de conformidade igual ou superior a $85 \%$ conforme o estabelecido pelas resoluções vigentes no país, com a finalização do estudo.

Com o término da pesquisa, designou-se um dos servidores, devidamente qualificado, como responsável por todas as ações de gestão ambiental da unidade, bem como o PGRSS. Acredita-se que os processos implantados tenham-se condições de se perpetuarem, com possibilidade destes serviços tornarem referência dentro da rede municipal de saúde de Palmas, como instituições que cumprem com expertise estes requisitos legais e de boas práticas.

Conclui-se que o envolvimento dos servidores, a partir das qualificações desenvolvidas, com os processos englobados pelo gerenciamento dos resíduos, foi decisivo para o sucesso da pesquisa e a consolidação destas práticas nas unidades.

Este estudo também mostrou que para realizar a grande maioria das 
adequações nestas unidades, não foram necessários investimentos de recursos financeiros, e sim um aprimoramento da capacidade gerencial de cada serviço como um esforço para atender as normas legais vigentes no país.

O gerenciamento do manejo dos resíduos de saúde e sua redução, não é apenas uma questão de atender a legislação e reduzir custos, mas uma questão ética de responsabilidade socioambiental. Se houver uma prática correta de manejo dos RSS, haverá benefícios aos usuários dos serviços de saúde, à comunidade, ao meio ambiente e à segurança dos trabalhadores da área de saúde.

\section{REFERÊNCIAS}

AYRES, Manuel; AYRES Manuel Jr.; AYRES, Daniel Lima; SANTOS, Alex de Assis dos. BioEstat: Aplicações estatísticas nas áreas das ciências biomédicas. Belém (Pará):Instituto Mamiraua, 2007.

BRASIL, Ministério da Saúde. Agência Nacional de Vigilância Sanitária. Manual de gerenciamento de resíduos de serviços de saúde. Brasília-DF, 2006.

Ministério da Saúde. Secretaria de Atenção à Saúde. Departamento de Atenção Especializada. Manual para Elaboração do Plano de Gerenciamento de Resíduos dos Serviços de Hematologia e Hemoterapia. Brasília-DF, 2012a.

, Ministério da Saúde. Agência Nacional de Vigilância Sanitária. Manual de Segurança do Paciente em serviços de Saúde: Limpeza e Desinfecção de Superfícies. Brasília-DF, 2012b.

Ministério das Cidades. Sistema Nacional de Informações sobre Saneamento - SNIS. Diagnóstico do Manejo de Resíduos Sólidos Urbanos de 2014. Brasília-DF, 2016.

. Ministério da Saúde. Agência Nacional de Vigilância Sanitária-ANVISA. Resolução da Diretoria Colegiada n`306 de 07 de Dezembro de 2004. Dispõe sobre o Regulamento Técnico para o Gerenciamento de Resíduos de Serviços de Saúde. Brasília, DF, 2004.

Ministério do Meio Ambiente, Conselho Nacional do Meio Ambiente CONAMA. Resolução da Diretoria Colegiada $n^{\circ} 358$ de 29 de Abril de 2005. Dispõe sobre o tratamento e a disposição final dos resíduos de serviços de saúde e dá outras providencias. Brasília, DF, 2005. 
Ministério do Trabalho e Emprego. Norma Regulamentadora 32 Segurança e Saúde no Trabalho em Serviços de Saúde. Brasília, DF, 2005.

Lei 12.305, de 2 de agosto de 2010. Institui a Política Nacional de Resíduos Sólidos. Diário Oficial da República Federativa do Brasil, Poder Executivo, Brasília, DF, 03 ago. 2010. Seção 1, p.3.

CAETANO, M.O. \& GOMES, L.P. Proposta de plano de gerenciamento de resíduos de serviços de saúde para o hospital Beneficência Portuguesa - Porto Alegre - RS. Estudos Tecnológicos, São Leopoldo-RS, v. 2, n. 2, p. 99-112, jul. dez. 2013.

IBGE - Instituto Brasileiro de Geografia e Estatística. Estimativas da população residente no Brasil e unidades da federação com data de referência em 1을 julho de 2014. Disponível em: ftp://ftp.ibge.gov.br/Estimativas_de_Populacao/Estimativas_2014/estimativa_dou_20 14.pdf, acessado em 20/06/2016.

JACOBI, P. R.; BESEN, G. R. Gestão de resíduos sólidos em São Paulo: desafios da sustentabilidade. Estudos avançados. vol. 25, n.71, p. 135-158. ISSN 0103-4014. São Paulo, 2011.

LORENTZ, Juliana Ferreira. Aplicação de recursos de roteirização e redes na coleta e transporte de resíduos de serviços de saúde. 2011. 68 f. Dissertação (Mestre em Análise e Modelagem de Sistemas Ambentais) - Instituto de Geociências, Universidade Federal de Minas Gerais, Belo Horizonte-MG, 2011.

PEREIRA, M.S. et al. Gerenciamento de resíduos em unidades não hospitalares de urgência e emergência. Revista Latino-Americana. Enfermagem. jan.-fev. 2013.

PEREIRA, S.S.; Resíduos de serviço de saúde: definição, classificação e legislação. Rev. Âmbito Jurídico, Rio Grande do Sul, XIV, n. 93, out 2011.

PREFEITURA MUNICIPAL DE PALMAS. Serviços de Saúde Oferecidos no Município: rede municipal de saúde em Palmas. Disponível em: <http://www.palmas.to.gov.br/servicos/servicos-de-saude-oferecidos-nomunicipio/200/>. Acesso em: 04 de agosto de 2014.

SILVA, I. T. S; BONFADA, D. Resíduos Sólidos de Serviços de Saúde e Meio Ambiente: Percepção da Equipe De Enfermagem. REVERENE - Revista da Rede de enfermagem do Nordeste, Caicó - RN, 2012.

STEHLING, M. C., et al. Gestão de resíduos com risco biológico e perfurocortantes: Conhecimento de estudantes de graduação das áreas biológicas e da saúde da Universidade Federal de Minas Gerais. Revista Mineira de Enfermagem (REME), Belo Horizonte. p. 594-600, jul-set. 2013. 
Recebido em: 22/08/2016

Aprovado em: 28/08/2017 